\title{
Monte Carlo Simulation of Thermal Radiation from Relativistic Media
}

\author{
Sanshiro Shibata ${ }^{1}$ and Nozomu Tominaga ${ }^{1,2}$ \\ ${ }^{1}$ Department of Physics, Konan University \\ ${ }^{2}$ Kavli Institute for the Physics and Mathematics of the Universe, University of Tokyo, 5-1-5 \\ Kashiwanoha, Kashiwa, Chiba 277-8583, Japan \\ E-mail: d1221001dcenter.konan-u.ac.jp
}

\begin{abstract}
Recently, thermal radiation from gamma-ray burst (GRB) jets attracts attention as the origin of the GRB prompt emission. In order to treat the thermal radiation from GRB jets properly, both the radiative transfer in the jets and the complicated inner structures of the jets should be taken into account. We developed a numerical code to calculate the radiative transfer in relativistic jets, based on the Monte-Carlo method. The code enables us to calculate the Compton scatterings by energetic electrons as a postprocessing in relativistic media with complicated structures obtained by $2 \mathrm{D}$ relativistic hydrodynamical calculations. In this paper, we investigated the influence of the location of the photon injection site on a GRB spectrum of the location. We show that the GRB spectrum has a higher peak energy for the deeper photon injection site.
\end{abstract}

Gamma-Ray Bursts 2012 Conference -GRB2012,

May 07-11, 2012

Munich, Germany 


\section{Introduction}

Recently, thermal radiation from gamma-ray burst (GRB) jets attracts attention as the origin of the GRB prompt emission because of high conversion efficiency to radiation and the fact that some GRBs show thermal components, e.g.[四, in the spectra of the prompt emission.

Hence thermal radiation from GRB jets have been investigated in the previous studies, e.g. []ㅣ [目][可]. The authors calculated the light curves and spectra only by superposing blackbody radiation emitted from the photosphere, i.e. from the surface of $\tau=1$ where $\tau$ is optical depth for electron scattering.

However, the observed photons should be generated in the inner layer with $\tau>1$. In order to treat the thermal radiation from GRB jets properly, both the radiative transfer in the jets and complex structures of the jets and cocoon should be taken into account.

In this paper, we present results of the calculation of radiative transfer in the relativistic jets. The hydrodynamical structure is derived by $2 \mathrm{D}$ special relativistic hydrodynamical calculation of the jet propagating in the Wolf-Rayet star. We investigated the influence on a GRB spectrum of the location of the photon production site. In section 2, we describe the initial conditions of hydrodynamical calculation whose result is used for hydrodynamical background and describe our radiative transfer code. In section 3, we present the results of the radiative transfer calculation.

\section{Method}

First, we calculated the jet structure with $2 \mathrm{D}$ relativistic hydrodynamical code with newtonian self gravity []]. A computational domain ranges from $r_{\text {in }}=10^{9} \mathrm{~cm}$ and is captured by 600 logalithmical grids in the r-direction and 150 uniform grids in the $\theta$-direction. The relativistic jet is injected by imposing boundary conditions at $r_{\text {in }}$. The conditions are the total jet luminosity $L_{\text {jet }}=5.3 \times 10^{50} \mathrm{erg} \mathrm{s}^{-1}$, the initial Lorentz factor $\Gamma_{0}=5$, the half opening angle of the jet $\theta_{0}=10^{\circ}$, and the thermal to total energy density ratio $f_{\text {th }}=0.9925$ which corresponds to the conditions of specific internal energy $\varepsilon_{0} / c^{2}=80$. A $13 \mathrm{M}_{\odot}$ Wolf-Rayet star with the main-sequence mass of $40 \mathrm{M}_{\odot}$ is used for the progenitor star [6].

The radiative transfer in the complex hydrodynamical background is calculated using MonteCarlo method. The photons are injected at the surface of $\tau=\tau_{\text {inj }}$ on the snapshot of the above calculation at $t=40 \mathrm{~s}$. Here, we adopt the optical depth for Thomson scattering defined as [ [ $]$

$$
\tau=\int_{z}^{\infty} \sigma_{\mathrm{T}} \Gamma(1-\beta \cos \theta) n_{\mathrm{e}} d z
$$

and take $\tau_{\text {inj }}=1,5,7$, and 10 . The energies of injected photons are determined as to obey the Planck distribution with its local plasma temperatures. The propagation directions of the injected photons are isotropic in the fluid rest frame.

After the injection, the photons propagate through the jet with some scatterings by electrons and freely escape from the jet when the optical depth decreases to one or so. The Compton scattering by the electrons is treated as follow: (1)the "4-momentum" of a photon in the observer frame is Lorentz transformed twice, i.e. into the fluid rest frame and the electron rest frame, (2)the "4momentum" of a photon after Compton scattering with Klein-Nishina cross section in electron rest 


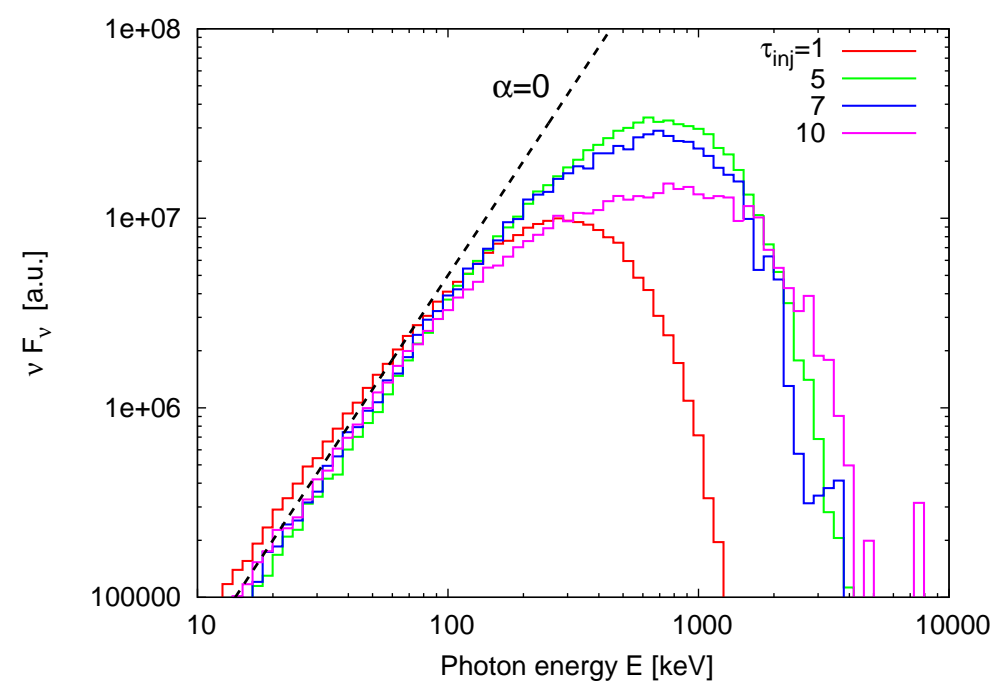

Figure 1: The emergent spectra for the models with $\tau_{\mathrm{inj}}=1$ (red), 5 (green), 7 (blue), and 10 (magenta).

frame is calculated using random numbers at each scattering indexes, (3)the "4-momentum" in the observer frame can be obtained doing inverse Lorentz transformation twice again.

We collect the photons escaped along the jet axis and construct the emergent energy spectrum observed by the on-axis observer.

\section{Results and Discussion}

Figure 1 shows the emergent spectra for the models with $\tau_{\text {inj }}=1,5,7$, and 10 .

The peak energy of the emergent spectrum is higher for larger $\tau_{\text {inj. }}$. The Band function parameter $\alpha$ (low energy index of the photon counts) is $\alpha=0$ for all models. This index is harder than observational typical value, $\alpha=-1$, but softer than Planck function, i.e. Rayleigh-Jeans tail which has $\alpha=1$.

When the optical depth is large, the photons are coupled with electrons via Compton scattering and cool adiabatically. However, as the optical depth decreases to moderate value, e.g. $\tau<10$, adiabatic cooling no longer works efficiently, e.g. [ $[\mathbb{8}][$ [ $]$. Therefore, if the photons are injected at $\tau \gg 10$, the plasma temperatures at the injection sites may not affect the emergent spectra because of the efficient adiabatic cooling. On the other hand, if the photons are injected at moderate optical depth, the plasma temperatures at the injection sites affect the emergent spectra because the energies of injected photons are underestimated in such cases and the peak energies for the model with larger $\tau_{\text {inj }}$ become higher. Recent study shows that the peak energy forms at the radius with the optical depth $\tau>30$ [ए]]. So we have to calculate with much higher $\tau_{\text {inj }}$ than that are taken here.

In this article, we used a snapshot of the jet structure at $t=40 \mathrm{~s}$. However, the time dependence of the jet structure should affect the emerging spectrum. The time dependent calculation will be done by the forthcoming papers. 


\section{Acknowledgements}

We thank to Hajime Susa for his helpful discussions. This research was supported, in part, by a grant from the Hayakawa Satio Fund awarded by the Astronomical Society of Japan.

\section{References}

[1] F. Ryde, M. Axelsson, B. B. Zhang, et al. Identification and Properties of the Photospheric Emission in GRB090902B, ApJL 709 (2010) L172

[2] D. Lazzati, B. J., Morsony, M. C. Begelman, Very High Efficiency Photospheric Emission in Long-duration $\gamma$-ray Bursts, ApJL 700 (2009) L47

[3] A. Mizuta, S. Nagataki, J. Aoi, Thermal Radiation from Gamma-Ray Burst Jets, ApJ 732 (2011) 26

[4] H. Nagakura, H. Ito, K. Kiuchi, S. Yamada, Jet Propagation, Breakouts, and Photospheric Emission in Collapsing Massive Progenitors of Long-duration Gamma-Ray Bursts, ApJ 731 (2011) 80

[5] N. Tominaga, Aspherical Properties of Hydrodynamics and Nucleosynthesis in Jet-induced Supernovae, ApJ 690 (2009) 526

[6] H. Umeda \& K. Nomoto, Variations in the Abundance Pattern of Extremely Metal-Poor Stars and Nucleosynthesis in Population III Supernovae, ApJ 619 (2005) 427

[7] M. A. Abramowicz, I. D. Novikov, B. Paczynski, The Appearance of Highly Relativistic, Spherically Symmetric Stellar Winds, ApJ 369 (1991) 175

[8] A. Pe'er, Temporal Evolution of Thermal Emission from Relativistically Expanding Plasma, ApJ 682 (2008) 463

[9] A. M. Beloborodov, Radiative Transfer in Ultrarelativistic Outflows, ApJ 737 (2011) 68

[10] A. M. Beloborodov, Regulation of the Spectral Peak in Gamma-Ray Bursts (2012) [arXiv:1207.2707] 